\title{
Two-Pulse Phase Modulation Based Decoupling Schemes for Removing Heteronuclear Spin Interactions in the NMR Spectroscopy of Isotropic Systems: An Analysis Using Experiments and Numerical Simulations
}

\author{
Cyril Augustine \\ Department of Chemistry, St. Berchmans College Campus, Mahatma Gandhi University, Kottayam, Kerala 686101, India
}

Correspondence should be addressed to Cyril Augustine; cyrilaug@yahoo.com

Received 29 January 2014; Accepted 7 April 2014; Published 15 May 2014

Academic Editor: Guang Zhu

Copyright (C) 2014 Cyril Augustine. This is an open access article distributed under the Creative Commons Attribution License, which permits unrestricted use, distribution, and reproduction in any medium, provided the original work is properly cited.

Heteronuclear spin interactions in solids and anisotropic liquids can be removed by phase modulated decoupling sequences with frequency sweep, such as $\mathrm{SW}_{\mathrm{f}}$-TPPM and its variants. The performance of these sequences in isotropic spin systems with regard to the experimental parameters, phase angle, and decoupler offset is presented here. A comparison is made with other commonly used heteronuclear decoupling schemes in liquids, namely, WALTZ-16, GARP, and MLEV. Also, the trajectories of nuclear magnetization vector of abundant nuclei in a simple spin system during TPPM and $\mathrm{SW}_{\mathrm{f}}$-TPPM decoupling sequences are traced out using computer simulations.

\section{Introduction}

The interactions between different types of nuclear spins in heteronuclear spin systems lead to the splitting of NMR peaks. Unlike anisotropic liquids and solids, where both dipole-dipole and J-coupling prevail, systems of isotropic liquids show only $J$-coupling because the secular parts of the intramolecular and intermolecular dipole-dipole couplings average to zero due to rapid tumbling motion of the molecules [1]. Improvement in the spectral quality of rare spins requires decoupling of the abundant spins from the former during the acquisition of NMR signals and needs suitable radiofrequency irradiation schemes [2]. Efficient decoupling over the entire band of proton resonance frequencies is an important factor in determining the resolution and sensitivity in ${ }^{13} \mathrm{C}$ spectroscopy $[3,4]$.

The decoupling sequence called swept-frequency twopulse phase modulation or $\mathrm{SW}_{\mathrm{f}}$-TPPM is one of the commonly used heteronuclear dipolar decoupling schemes proposed for application in solid-state nuclear magnetic resonance [5]. It was obtained by varying the pulse duration of another phase-switched decoupling sequence, two-pulse phase modulation (TPPM), where radiofrequency pulses of length $\tau_{p}$ alternating between two phases separated by an angle $\varphi$ were used [6]. $\mathrm{SW}_{\mathrm{f}}$-TPPM works admirably for heteronuclear dipolar decoupling in solid samples [5] and also in liquid crystals [7]. Currently, there exists a family of sequences in the literature [8-14] based on $\mathrm{SW}_{\mathrm{f}}$-TPPM for heteronuclear dipolar decoupling in solids and liquid crystals.

As already remarked, the TPPM and $\mathrm{SW}_{\mathrm{f}}$-TPPM family of sequences were originally developed for heteronuclear dipolar decoupling in solid-state NMR. Although the underlying principles of decoupling in isotropic and anisotropic systems are different, it would be interesting to know the behavior of these decoupling sequences in isotropic liquids and to understand their efficiency in removing scalar heteronuclear interactions. The spin dynamics during these sequences can be explored easily in the liquid state because of the smaller interaction strengths and the absence of many body effects.

In this report, an attempt is made to explore the efficiency of $\mathrm{SW}_{\mathrm{f}}$-TPPM for heteronuclear decoupling in isotropic systems and compare the results with some commonly used broadband decoupling sequences in liquid state NMR, MLEV 
[15-17], WALTZ-16 [18, 19], and GARP [20]. Besides the TPPM sequence, the efficiencies of the parent $S_{\mathrm{f}}$-TPPM and some other phase modulated swept-frequency decoupling schemes derived from $\mathrm{SW}_{\mathrm{f}}$-TPPM by decreasing the number of TPPM blocks, namely, $\mathrm{SW}_{\mathrm{f}}-\mathrm{TPPM}-7$ [13], $\mathrm{SW}_{\mathrm{f}^{-}}$ TPPM-6, SW $\mathrm{f}_{\mathrm{f}}$-TPPM-5, and $\mathrm{SW}_{\mathrm{f}}$-TPPM-4, which contains 7, 6, 5, and 4 TPPM blocks, respectively, are compared. The rationale in decreasing the number of TPPM blocks was to bring down the length of decoupling sequence with as little effect on the efficiency and resolution. The efficiency of decoupling was determined in each of these sequences on varying the proton offsets and phase angles.

It can be observed in the literature that the efficiency of a decoupling sequence can be improved by combining different versions of the primitive cycle into extended supercycles in which some of the residual pulse imperfections are compensated [15, 16]. Examples include MLEV [15-17], WALTZ [18, 19], SPARC [21], and SPINAL [22] sequences. The first two are used in isotropic liquids for removing scalar couplings and the remaining two sequences are designed for solids and liquid crystals to remove mainly the heteronuclear dipolar couplings. The effect of supercycling in the swept frequency phase modulated sequences is already reported for solid-state and liquid crystal NMR [13, 14]. Here, the role of supercycling in the swept frequency phase modulated sequences is analyzed in isotropic liquids using experiments and numerical calculations.

\section{Materials and Methods}

2.1. Decoupling Schemes. The scheme for two-pulse phase modulation (TPPM) [12] consists of two pulses of equal length $\tau$ with phases $\varphi$ and $-\varphi$. It can be denoted as $\left[\tau_{\varphi} \tau_{-\varphi}\right]$. Generally it appears in the forms $165_{-}\{10\}$ and $165_{-}\{-10\}$ where 165 implies RF pulse angle and 10 its phase angle in degrees. It should be noted that, for effective performance of TPPM, $\tau$ and $\varphi$ are to be optimized.

The $\mathrm{SW}_{\mathrm{f}}$-TPPM sequence [5] was formulated with TPPM as the building block. It is done by varying the pulse length in TPPM in a tangential way. The sequence can be denoted as $\left\{\begin{array}{lll}0.78 \tau_{\varphi} & 0.78 \tau_{-\varphi}\end{array}\right]\left[\begin{array}{lll}0.86 \tau_{\varphi} & 0.86 \tau_{-\varphi}\end{array}\right]\left[\begin{array}{lll}0.94 \tau_{\varphi} & 0.94 \tau_{-\varphi}\end{array}\right]\left[\begin{array}{ll}0.96 \tau_{\varphi} \\ \hline\end{array}\right.$ $\left.0.96 \tau_{-\varphi}\right]\left[\begin{array}{lll}0.98 \tau_{\varphi} & 0.98 \tau_{-\varphi}\end{array}\right]\left[\begin{array}{l}\tau_{\varphi} \tau_{-\varphi}\end{array}\right]\left[\begin{array}{lll}1.02 \tau_{\varphi} & 1.02 \tau_{-\varphi}\end{array}\right]\left[\begin{array}{ll}1.04 \tau_{\varphi} \\ 0\end{array}\right.$ $\left.\left.1.04 \tau_{-\varphi}\right]\left[\begin{array}{lll}1.06 \tau_{\varphi} & 1.06 \tau_{-\varphi}\end{array}\right]\left[\begin{array}{lll}1.14 \tau_{\varphi} & 1.14 \tau_{-\varphi}\end{array}\right]\left[\begin{array}{lll}1.22 \tau_{\varphi} & 1.22 \tau_{-\varphi}\end{array}\right]\right\}$. There are 11 TPPM blocks in this sequence and their pulse lengths vary from $0.78 \tau$ to $1.22 \tau$ where $\tau$ is a normal $180^{\circ}$ pulse length. In the following discussions, this sequence is represented as $\mathrm{SW}_{\mathrm{f}}$-TPPM-11.

The multiplicative factors of pulse duration given in $\mathrm{SW}_{\mathrm{f}^{-}}$ TPPM-11 follow a tangential path and it is possible to make new variants with less number of TPPM blocks keeping the pulse length variation tangential. In this manner, four other sequences are designed. They include $\mathrm{SW}_{\mathrm{f}}-\mathrm{TPPM}-7$ [13], SW $\mathrm{S}_{\mathrm{f}}$-TPPM-6, $\mathrm{SW}_{\mathrm{f}}$-TPPM-5, and $\mathrm{SW}_{\mathrm{f}}$-TPPM-4, which contain 7, 6, 5, and 4 TPPM blocks, respectively. Their profiles are given in Figure 1. The numbers plotted on the ordinate in each profile are the multiplicative factors (denoted by $f$ ) that determine the corresponding profile. For example, the
$\mathrm{SW}_{\mathrm{f}}$-TPPM-7 [13] sequence can be designated as $\left\{\left[0.82 \tau_{\varphi}\right.\right.$ $\left.0.82 \tau_{-\varphi}\right]\left[\begin{array}{lll}0.90 \tau_{\varphi} & 0.90 \tau_{-\varphi}\end{array}\right]\left[\begin{array}{lll}0.98 \tau_{\varphi} & 0.98 \tau_{-\varphi}\end{array}\right]\left[\begin{array}{l}\tau_{\varphi} \tau_{-\varphi}\end{array}\right]\left[\begin{array}{ll}1.02 \tau_{\varphi} \\ 0\end{array}\right.$ $\left.\left.1.02 \tau_{-\varphi}\right]\left[1.10 \tau_{\varphi} 1.10 \tau_{-\varphi}\right]\left[1.18 \tau_{\varphi} 1.18 \tau_{-\varphi}\right]\right\}$. As the name implies there are 7 TPPM blocks in this sequence and their pulse lengths vary from $0.82 \tau$ to $1.18 \tau$. The sequences $\mathrm{SW}_{\mathrm{f}}-\mathrm{TPPM}$ $6, \mathrm{SW}_{\mathrm{f}}$-TPPM-5, and $\mathrm{SW}_{\mathrm{f}}$-TPPM-4 are similarly formulated. Even though $S_{\mathrm{f}_{\mathrm{f}}}$-TPPM-4 is the shortest sequence it still maintains a reasonable tangential sweep in the pulse length as shown in Figure 1.

For each of these sequences, the effectiveness of decoupling was analyzed as a function of the proton offset and phase angle, $\varphi$. Experiments were performed for phase angles $15^{\circ}$, $20^{\circ}, 25^{\circ}, 30^{\circ}, 35$, $^{\circ}$ and $40^{\circ}$ and the proton offset was varied from 2 to $12 \mathrm{ppm}$ with respect to TMS.

To analyze the effect of constructing supercycles on the efficiency of swept frequency phase modulated sequences, the parent $S_{\mathrm{f}_{\mathrm{f}}}$-TPPM-11 sequence was taken as $R$ and a supercycle was made to form $R R \bar{R} \bar{R}$ where $\bar{R}$ gives the phase inverted counterpart of $R[13,14]$. Likewise, supercycled versions of shorter $\mathrm{SW}_{\mathrm{f}}-\mathrm{TPPM}$ decoupling sequences $\mathrm{SW}_{\mathrm{f}^{-}}$ TPPM-7 and SW $\mathrm{S}_{\mathrm{f}}$-TPPM-4 also were constructed and their performance was studied.

2.2. Experimental. Proton decoupled ${ }^{13} \mathrm{C}$ experiments of benzene dissolved in acetone- $\mathrm{d}_{6}$ were performed on a BRUKER AV spectrometer at a frequency of $300 \mathrm{MHz}$ for proton. Eight scans were accumulated for each spectrum with $8 \mathrm{k}$ data points in a time of $0.542 \mathrm{~s}$. The FID was Fourier transformed after exponential multiplication with a $1 \mathrm{~Hz}$ damping function. The experiments were performed at a decoupler power corresponding to a $\pi / 2$ time of $87.75 \mu \mathrm{s}$, which corresponds to a decoupler nutation frequency of $2 \pi v_{1}=$ $2 \pi /(4 \times 87.75 \mu \mathrm{s})=17925 \operatorname{radians}^{-1}$ or $v_{1}=2.85 \mathrm{kHz}$. This RF power is typically used at our facility for all ${ }^{13} \mathrm{C}$ decoupling experiments. The ${ }^{1} \mathrm{H}$ decoupling pulse powers were calibrated with the DECP90 pulse program. The pulse sequence for DECP90 includes a $90^{\circ}{ }^{13} \mathrm{C}$ pulse, a delay set to $1 /\left[2 J\left({ }^{13} \mathrm{C},{ }^{1} \mathrm{H}\right)\right]$ for the evolution of antiphase ${ }^{13} \mathrm{C}-{ }^{1} \mathrm{H}$ magnetization, and a ${ }^{1} \mathrm{H}$ pulse whose duration is increased step by step, followed by ${ }^{13} \mathrm{C}$ detection. When the ${ }^{1} \mathrm{H}$ pulse length corresponds to a flip angle of $90^{\circ}$, a null in the detected signal can be observed.

The ${ }^{13} \mathrm{C}$ NMR spectrum of benzene with proton decoupling is measured as the decoupler radiofrequency offset was varied from 2 to $12 \mathrm{ppm}$ with respect to TMS. The proton resonance frequency is at $\sim 7.5 \mathrm{ppm}$ and the ${ }^{13} \mathrm{C}$ resonance appears at $\sim 128 \mathrm{ppm}$. Since the RF field strength is constant the bandwidth of the decoupling can be considered as a measure of its efficiency. In the figures, intensity of the decoupled line corresponding to the ${ }^{13} \mathrm{C}$ resonance of benzene is plotted with successive increments of offset frequencies.

The simulations required to understand and visualize the trajectories of nuclear magnetization vector corresponding to the decoupled nuclei in a simple spin system, benzene during various decoupling sequences, were carried out using a Bloch Simulator. 


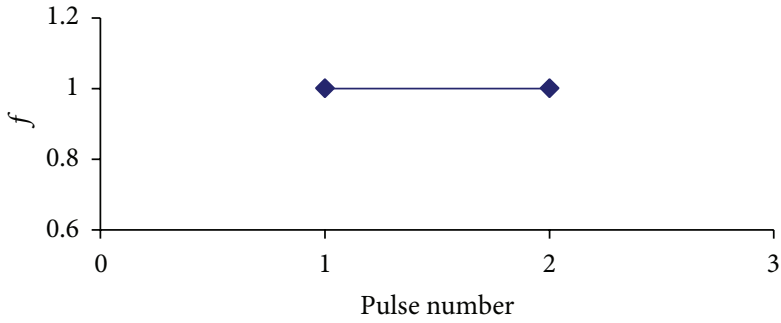

(a)

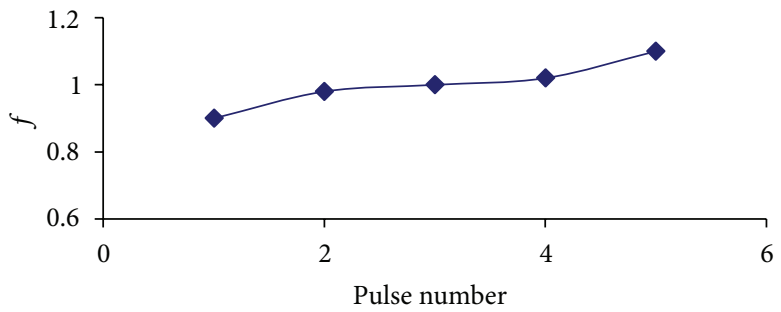

(c)

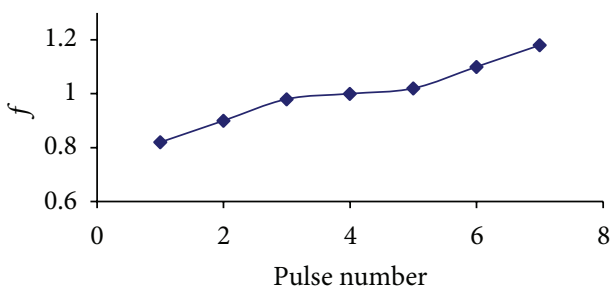

(e)

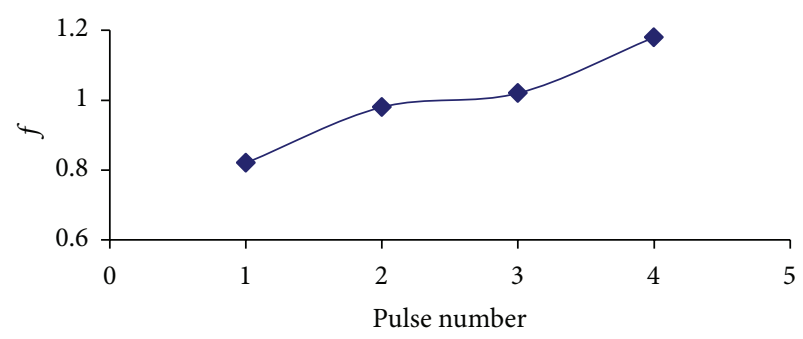

(b)

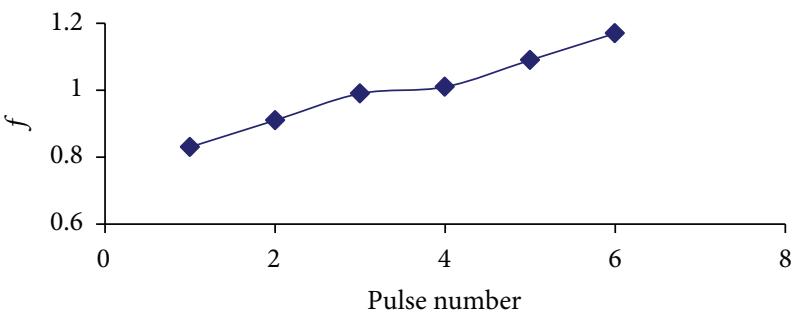

(d)

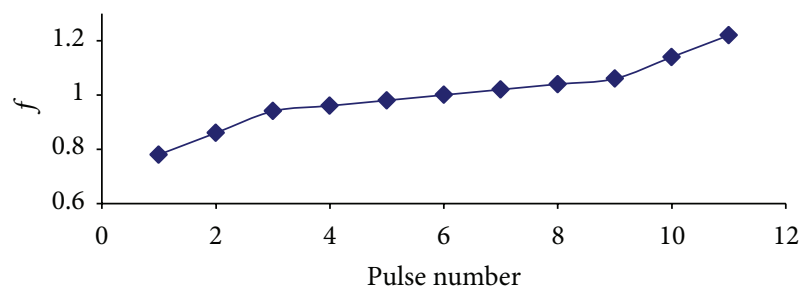

(f)

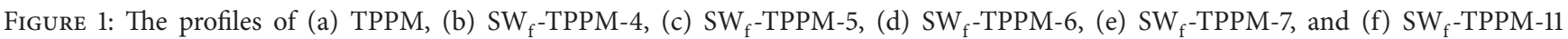
sequences. The pulse length, normalized with respect to the central pulse pair of various $S_{\mathrm{f}}$-TPPM sequences, is plotted against the number of the TPPM blocks in the corresponding decoupling sequence. The ordinate denotes the multiplicative factors that determine the corresponding profile.

\section{Results and Discussion}

The efficiency of various $\mathrm{SW}_{\mathrm{f}}$-TPPM sequences for heteronuclear decoupling in liquid state NMR is analyzed and the results are summarized below. Experiments were performed with the parent $\mathrm{SW}_{\mathrm{f}}$-TPPM-11 and its variants, $\mathrm{SW}_{\mathrm{f}}$-TPPM-7, $\mathrm{SW}_{\mathrm{f}}$-TPPM-6, SW $\mathrm{f}_{\mathrm{f}}$-TPPM-5, SW $\mathrm{f}_{\mathrm{f}}$ TPPM-4, and TPPM.

In all these experiments, a constant phase angle of $15^{\circ}$ is used with an RF power of $2.85 \mathrm{kHz}$. In TPPM, SW $\mathrm{f}_{\mathrm{f}}$ TPPM5 , and $\mathrm{SW}_{\mathrm{f}}$-TPPM-4 the decoupling bandwidth is smaller than for sequences with more number of elements as shown in Figure 2(a). Since the commonly used broadband decoupling sequences in liquid state NMR are MLEV, WALTZ, and GARP, it is appropriate to compare the efficiencies of TPPM and $\mathrm{SW}_{\mathrm{f}}$-TPPM sequences with them. Within the investigated proton offset range, there is almost no change in the intensities of the decoupled ${ }^{13} \mathrm{C}$ spectra of benzene during MLEV, WALTZ, or GARP decoupling as shown in Figure 2(b). This indicates that decoupling sequences designed specifically for liquid state applications outperform the TPPM and $\mathrm{SW}_{\mathrm{f}}$-TPPM sequences. From this evidence it is clear that TPPM and its variants are inappropriate for heteronuclear decoupling in isotropic systems. Significantly, this is an expected result because TPPM and its variants were designed for decoupling in solids where heteronuclear dipolar couplings dominate.

Experiments were conducted to study the effect of phase angles on the decoupling bandwidth in $\mathrm{SW}_{\mathrm{f}}$-TPPM sequences with $4,5,6,7$, and 11 TPPM elements. In each case, there exists an optimum phase angle which gives us maximum decoupling bandwidth. For instance, a phase angle of $20^{\circ}$ performs well in $\mathrm{SW}_{\mathrm{f}}$-TPPM-11 and $\mathrm{SW}_{\mathrm{f}}$-TPPM-5.In case of $S_{\mathrm{f}}$-TPPM-7, SW $\mathrm{f}_{\mathrm{f}}$-TPPM-6, and $\mathrm{SW}_{\mathrm{f}}$-TPPM-4, $25^{\circ}$ is the optimum phase angle. The results for $\mathrm{SW}_{\mathrm{f}}-\mathrm{TPPM}$ 11 and SW $_{\mathrm{f}}$-TPPM-7 are shown in Figures 3(a) and 4(a), respectively. In all cases the curves showing the effectiveness of decoupling oscillate with remarkable fluctuations in the intensities, rather than vary monotonically as the phase angle is increased, and show symmetrical depressions athigher values of the phase angles. But there are some points of decoupler offset, away from the on-resonance position which shows enhanced decoupling. For example, in case of $\mathrm{SW}_{\mathrm{f}^{-}}$ TPPM-11 sequence with a phase angle of $35^{\circ}$ the curve shows depressions in intensity at 5 and $10 \mathrm{ppm}$ in a symmetrical fashion whereas at 4 and $11 \mathrm{ppm}$ there is enhanced decoupling even if these points are away from the on-resonance position as depicted in Figure 3(a). A similar pattern can be observed in all the other sequences. 


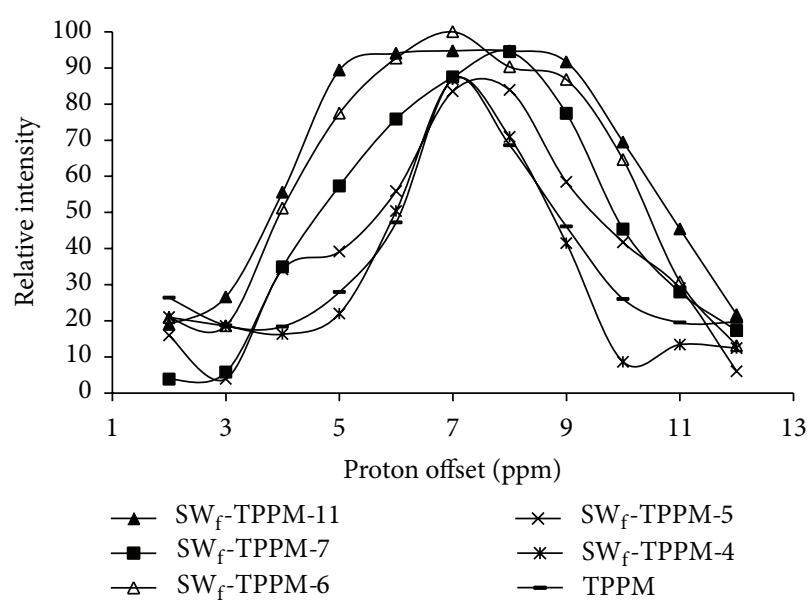

(a)

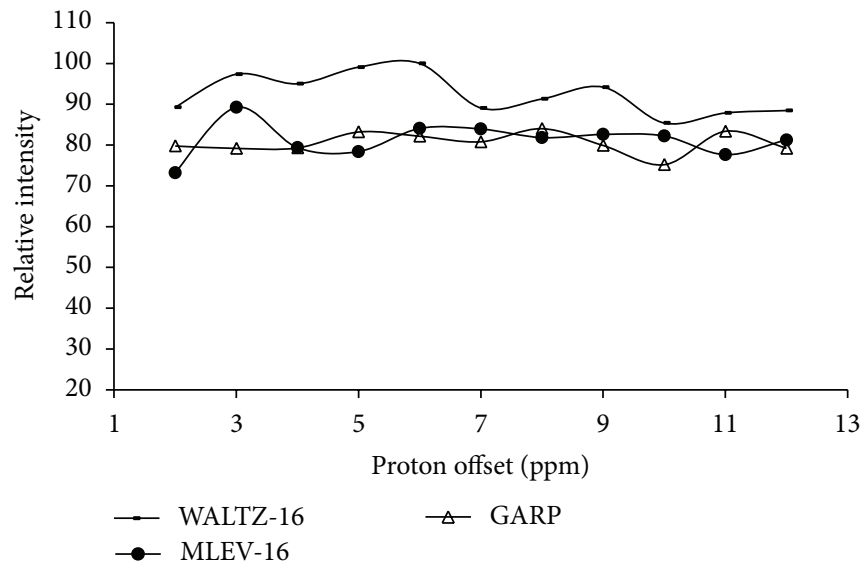

(b)

Figure 2: The relative intensities of the decoupled ${ }^{13} \mathrm{C}$ spectra of benzene at an RF power of $2.85 \mathrm{kHz}$ using (a) TPPM, SW $\mathrm{f}$-TPPM-11, 7, 6, 5, and 4 decoupling sequences at a phase angle of $15^{\circ}$ and (b) WALTZ-16, MLEV-16, and GARP decoupling sequences. The proton offset varies between 2 and $12 \mathrm{ppm}$ with respect to TMS.

In this situation let us think about some modifications to the existing sequences. An improvement in the effectiveness of decoupling at the points corresponding to the observed depressions of the curve is expected to give a larger decoupling bandwidth. Generally, improvement in the performance of a decoupling sequence can be achieved by uniting various combinations of the primitive cycle into extended supercycles. When we do so, some of the residual pulse imperfections are compensated. Decoupling experiments with the supercycled sequences show that there is significant improvement in its performance as shown in Figure 3(b). In this figure, the relative intensities of the decoupled ${ }^{13} \mathrm{C}$ spectra of benzene using the supercycled $\mathrm{SW}_{\mathrm{f}}$-TPPM-11 sequence with different phase angles are plotted as a function of the proton offset frequency. When the supercycled $\mathrm{SW}_{\mathrm{f}}$-TPPM11 sequence is used, the fluctuations in the intensities are suppressed and so is the oscillatory behavior as shown in Figure 3(b). Now the maximum efficiency of decoupling is at a phase angle of $40^{\circ}$. The performance of the supercycled sequence at a phase angle of $20^{\circ}$ which was the optimum value of the parent $\mathrm{SW}_{\mathrm{f}}$-TPPM-11 sequence is also good, but its effective decoupling bandwidth is lesser than the supercycled sequence with phase angle $40^{\circ}$ as depicted in Figure 3(b).

The case of supercycled sequence of $\mathrm{SW}_{\mathrm{f}}$-TPPM-7 is similarly giving a marginal improvement in the efficiency of decoupling as shown in Figure 4(b). This supercycled version also smoothens out the efficiency curves. The fluctuations in the intensities are suppressed and the oscillatory behavior is vanished as shown in the figure.

Thus, the effectiveness of $\mathrm{SW}_{\mathrm{f}}$-TPPM-11 and 7 sequences is marginally enhanced on supercycling, the oscillatory behavior is removed, and the fluctuations in the intensities are suppressed. The price one pays is that these sequences are relatively lengthy and hence new sequences which are shorter are to be preferred. In an attempt to achieve this objective, the efficiency of decoupling for the supercycled $\mathrm{SW}_{\mathrm{f}}-\mathrm{TPPM}-4$ sequence was studied as a function of phase angle. Even though the sequence fails to suppress the fluctuations in intensities and to give effective decoupling at all the phase angles analyzed, it performs relatively well at the phase angle of $40^{\circ}$. The illustrations are given in the supporting information (see supporting information in Supplementary Material available online at http://dx.doi.org/10.1155/2014/641473).

3.1. Simulation Studies. In the following sections, the trajectories of nuclear magnetization vector of the abundant nuclei in a simple spin system during TPPM and $\mathrm{SW}_{\mathrm{f}}$-TPPM decoupling are traced out on the Bloch sphere and compared with those obtained from WALTZ-16, MLEV, and GARP decoupling sequences which are commonly used in liquids for removing heteronuclear couplings. Finally, an attempt has been done to interpret the efficiency of decoupling in correlation with the trajectories of proton magnetization vectors during the implementation of the corresponding decoupling sequences.

Figure 5(a) shows the trajectory of proton magnetization vector on the Bloch sphere subjected to TPPM decoupling with a phase angle of $15^{\circ}$ at a radiofrequency offset corresponding to the proton resonance in a simple spin system, benzene. The magnetization vector starts from $+z$ axis, traverses a path downwards over the sphere, and reaches the $-z$-axis as shown. From $-z$, it follows a trajectory upwards over the opposite surface of the Bloch sphere and finally reaches at the initial position. Figure 5(b) depicts the time evolution of nuclear magnetization vector during TPPM decoupling with different offsets in increments of $200 \mathrm{~Hz}$ from the resonance position viewed from the top of the Bloch sphere through $z$-axis. In all cases the trajectories are almost the same and terminate near $+z$-axis. The set of magnetization trajectories induced by TPPM sequences at the given power of $2.85 \mathrm{kHz}$ shows that, in case of higher values of $\Delta B / B_{2}$, where $\Delta B$ is the offset range and $B_{2}$ is the decoupler 


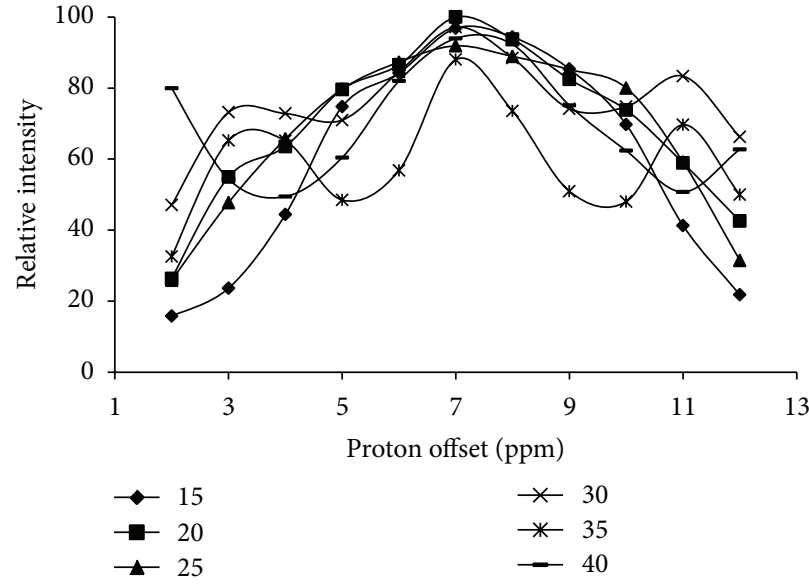

(a)

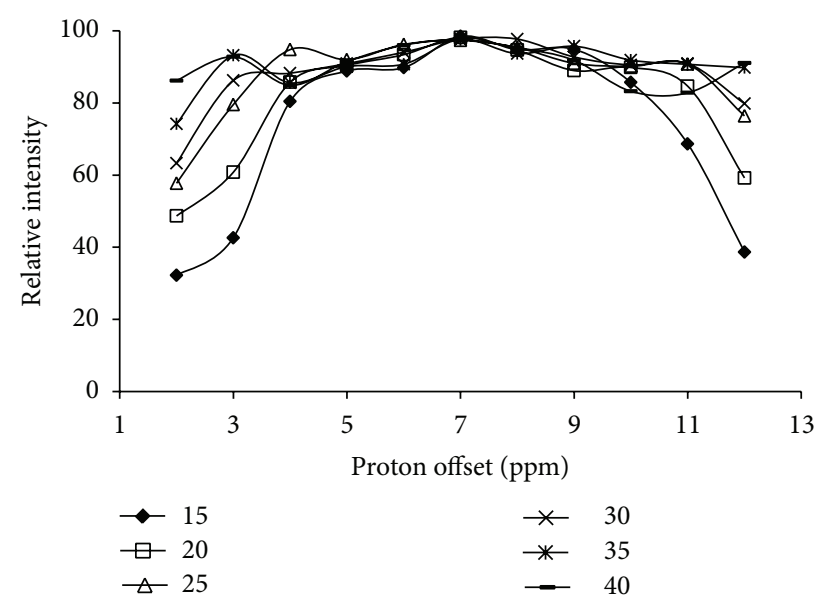

(b)

Figure 3: The relative intensities of the decoupled ${ }^{13} \mathrm{C}$ spectra of benzene at an RF power of $2.85 \mathrm{kHz}$ using (a) SW $\mathrm{f}_{\mathrm{f}}$-TPPM-11 and (b) its supercycled version. Phase angles of $15,20,25,30,35$, and $40^{\circ}$ are used. The proton offset varies between 2 and 12 ppm with respect to TMS.

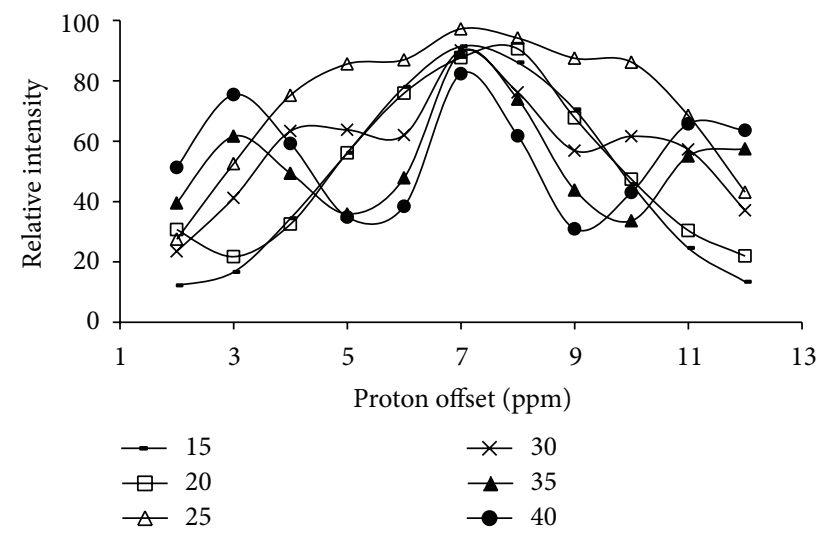

(a)

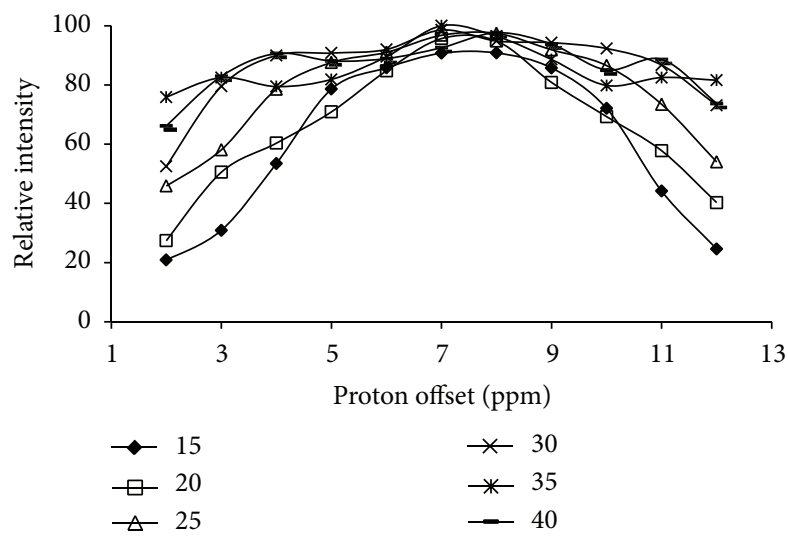

(b)

FIgure 4: The relative intensities of the decoupled ${ }^{13} \mathrm{C}$ spectra of benzene at an RF power of $2.85 \mathrm{kHz}$ using (a) $\mathrm{SW}$ - $\mathrm{TPPM}-7$ and $(\mathrm{b})$ its supercycled version. Phase angles of $15,20,25,30,35$, and $40^{\circ}$ are used. The proton offset varies between 2 and 12 ppm with respect to TMS.

field strength, increased effective field changes the trajectories and the termini start to drift from the $+z$-axis which was the terminating point at the resonance condition. The trajectories of the magnetization vector during TPPM decoupling with different phase angles resulted in similar observations.

Figure 6(a) is for $\mathrm{SW}_{\mathrm{f}}$-TPPM-11 decoupling sequence with a phase angle of $15^{\circ}$ at radiofrequency offset corresponding to the proton resonance in the same spin system. The magnetization vector starts from $+z$-axis and traverses a path over the Bloch sphere defined by the 11 pulse pairs. It does not come back to the initial position and ends near the equator of the sphere as shown with the circle. The diagram Figure 6(b) also depicts the time evolution of nuclear magnetization vector corresponding to the abundant nuclei during $\mathrm{SW}_{\mathrm{f}}$-TPPM-11 decoupling. Here, the proton offsets are varied with increments of $50 \mathrm{~Hz}$ from the resonance position at an RF power of $2.85 \mathrm{kHz}$. The region where the trajectories terminate is shown with an ellipse. For each value of the offset, the position at which the trajectory ends is different.

Now, we analyze the time evolution of nuclear magnetization vector for the supercycled sequences by taking the example of $\mathrm{SW}_{\mathrm{f}}$-TPPM-4. This supercycled sequence is a representative of the behavior shown by all other supercycled sequences and is depicted here because the crowded Bloch sphere in the other cases prevents drawing meaningful conclusions. Figure 7(a) depicts the simulated trajectory of nuclear magnetization vector of the abundant nuclei during $\mathrm{SW}_{\mathrm{f}}$-TPPM-4 decoupling with different offsets applying increments of $50 \mathrm{~Hz}$ from the resonance position. A phase angle of $40^{\circ}$ is used at a decoupling power of $2.85 \mathrm{kHz}$. The magnetization vector starts from $+z$ axis-and traverses a path over the Bloch sphere and does not return to the initial position, ending up near the equator of the sphere as shown. The termini drift continuously as a function of proton offset. 


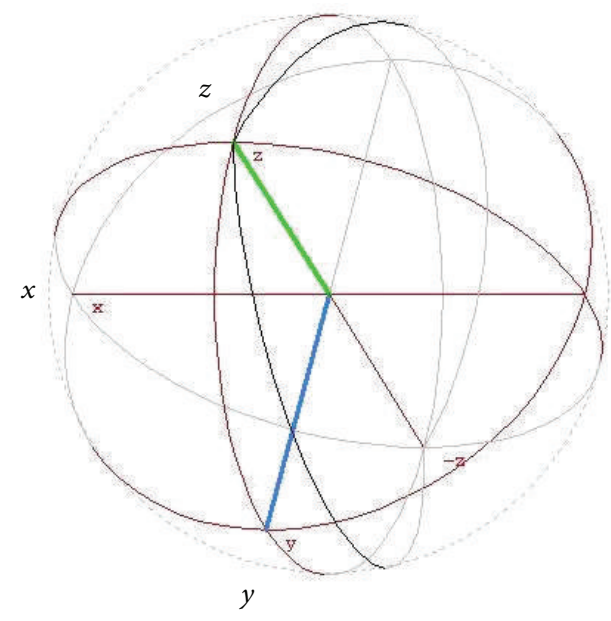

(a)

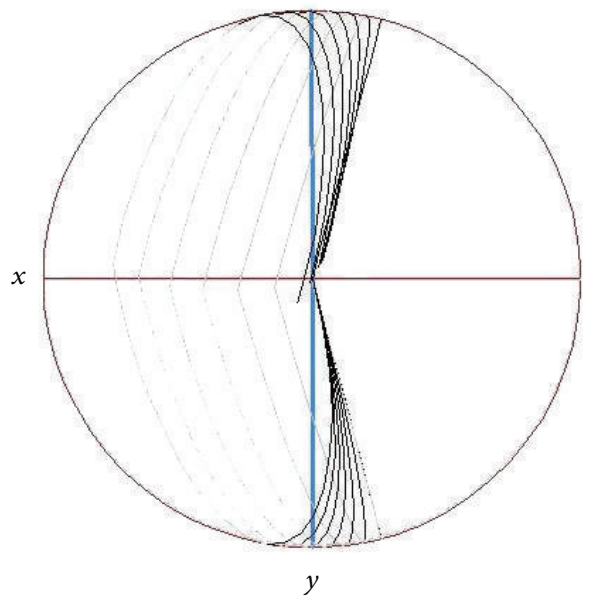

(b)

FIGURE 5: The simulated trajectory of proton magnetization vector during (a) TPPM decoupling at the resonance position and (b) TPPM with different offsets of $200 \mathrm{~Hz}$ increments from the resonance position (view from $z$-axis). A phase angle of $15^{\circ}$ is used with a decoupling power of $2.85 \mathrm{kHz}$.

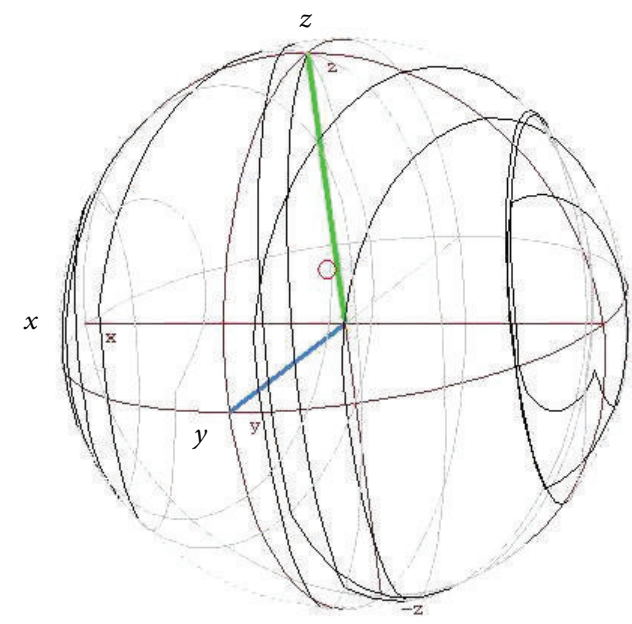

(a)

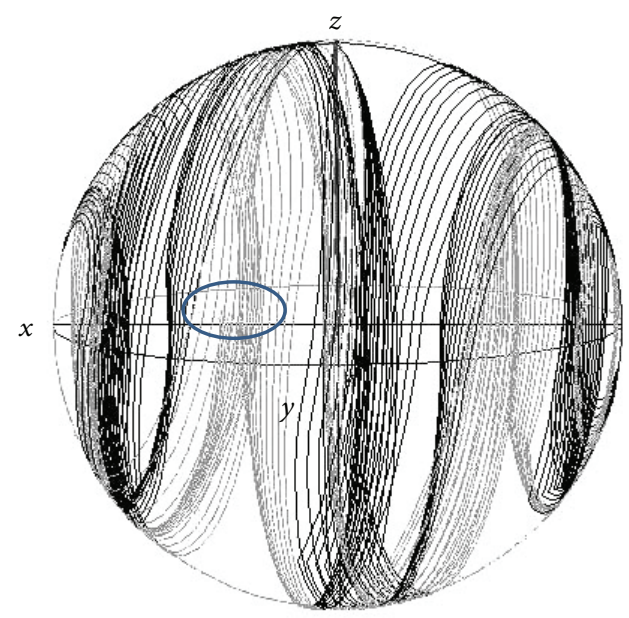

(b)

FIGURE 6: The simulated trajectory of proton magnetization vector during (a) $\mathrm{SW}_{\mathrm{f}}-\mathrm{TPPM}-11$ at the resonance position and (b) $\mathrm{SW}_{\mathrm{f}}$-TPPM-11 with different offsets of $50 \mathrm{~Hz}$ increments from the resonance position. The termini are marked. A phase angle of $15^{\circ}$ is used with a decoupling power of $2.85 \mathrm{kHz}$.

But, for the supercycled $\mathrm{SW}_{\mathrm{f}}$-TPPM-4, the termini of all the trajectories end at the $+z$-axis as shown in Figure 7 (b).

\subsection{Trajectories of Proton Magnetization Vector during $S W_{f^{-}}$} TPPM Decoupling: A Comparison with MLEV, WALTZ, and GARP. The group of proton magnetization trajectories, given by $\mathrm{SW}_{\mathrm{f}}$-TPPM sequences at the implemented power level of $2.85 \mathrm{kHz}$, tells that the increased effective field in case of higher values of $\Delta B / B_{2}$ changes the trajectories in the parent $S W_{f}$-TPPM sequences and the termini are drifting as a function of offset. But in the case of supercycled $S_{f_{f}}$-TPPM sequences, the trajectories of proton magnetization vector always end closer to the starting point at $+z$-axis which was the terminating point at the resonance condition. It should be noted that, at higher RF filed strength, $\mathrm{SW}_{\mathrm{f}}$-TPPM based sequences can give better results, but an RF power of $2.85 \mathrm{kHz}$ is sufficient for heteronuclear decoupling in isotropic systems as given by WALTZ-16, MLEV, and GARP schemes.

Figures $8(\mathrm{a})$ and $8(\mathrm{~b})$ show the trajectory of proton magnetization vector on the Bloch sphere subjected to heteronuclear decoupling using WALTZ-16 and MLEV-16, respectively. Different proton offsets with increments of $50 \mathrm{~Hz}$ from the resonance position are applied in a simple spin system, benzene at an RF power of $2.85 \mathrm{kHz}$. Unlike $\mathrm{SW}_{\mathrm{f}^{-}}$ TPPM based sequences, these trajectories always end in a position close to the $z$-axis, irrespective of the proton offsets, 


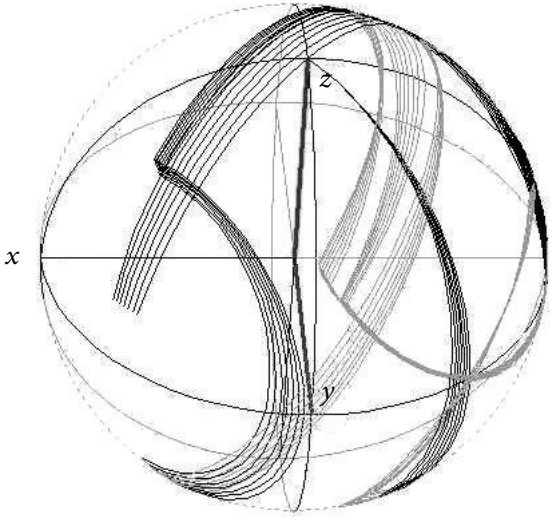

(a)

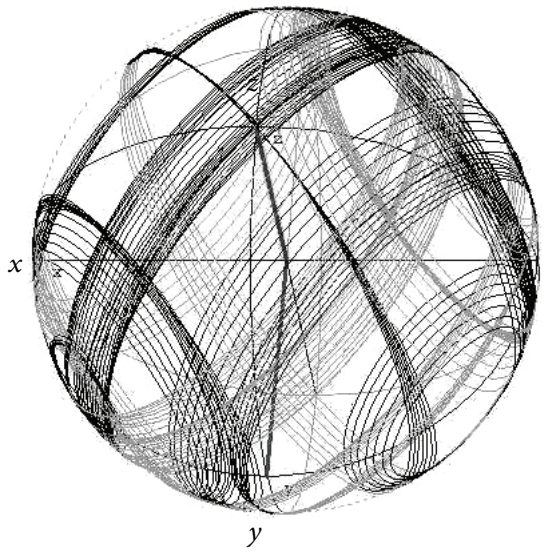

(b)

FIGURE 7: The simulated trajectory of proton magnetization vector with different offsets of $50 \mathrm{~Hz}$ increments from the resonance position during (a) $\mathrm{SW}_{\mathrm{f}}$-TPPM-4 and (b) supercycled $\mathrm{SW}_{\mathrm{f}}$-TPPM-4 decoupling schemes. A phase angle of $40^{\circ}$ is applied with a decoupling power of $2.85 \mathrm{kHz}$.

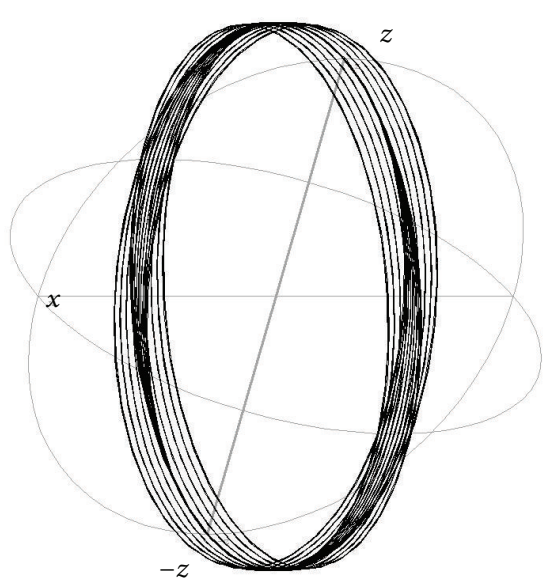

(a)

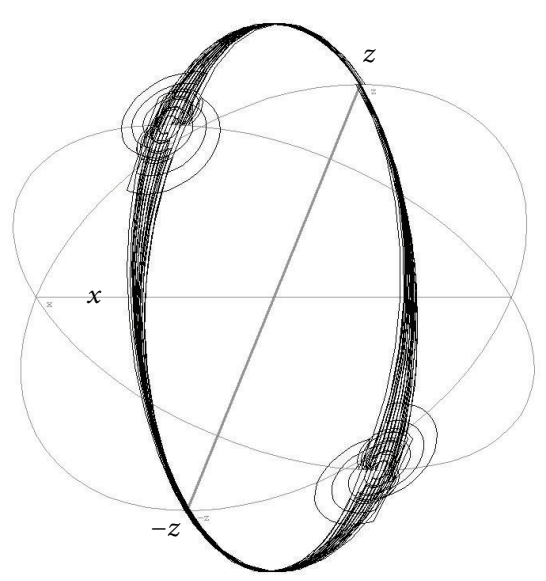

(b)

FIGURE 8: The simulated trajectory of proton magnetization vector with different offsets of $50 \mathrm{~Hz}$ increments from the resonance position during (a) WALTZ-16 and (b) MLEV-16 decoupling schemes. The decoupling power is $2.85 \mathrm{kHz}$.

and give efficient heteronuclear decoupling over a wide range of proton offsets.

3.3. Role of Phase Angle in $S W_{f}$-TPPM Sequences: Numerical Calculations. A careful analysis of the trajectories of proton magnetization vector during parent $\mathrm{SW}_{\mathrm{f}}$-TPPM sequences and their supercycled sequences reveals that, in the former case, the magnetization vector rotates according to the specified sequence from $+z$, for instance, as shown in Figures 6(a) and 8(a). Since the phase angle has a key role in determining the trajectory of proton magnetization vector during the sequence, the final position varies as a function of phase angle. The trajectory ends neither at $+z$ nor $-z$ during parent $\mathrm{SW}_{\mathrm{f}}$-TPPM sequences. But the trajectories traced by proton magnetization vector during supercycled $\mathrm{SW}_{\mathrm{f}}$-TPPM sequences always come back and end close to the $z$-axis irrespective of the phase angles. This is illustrated in the supporting information using supercycled $\mathrm{SW}_{\mathrm{f}}$-TPPM-4 and $\mathrm{SW}_{\mathrm{f}}$-TPPM-11 sequences (see supporting information in Supplementary Material).

\section{Conclusions}

The phase modulated decoupling sequences TPPM and $\mathrm{SW}_{\mathrm{f}^{-}}$ TPPM and the variants of the latter were used for heteronuclear decoupling in isotropic liquids. The effectiveness of decoupling was analyzed for these sequences and compared with the other commonly used decoupling sequences in isotropic liquids such as WALTZ-16, MLEV-16, and GARP. The broadband decoupling sequences that we use in liquid state outperform TPPM based sequences in removing heteronuclear couplings with minimum RF power. The efficiency of TPPM based sequences as a function of phase angles has been experimentally determined. Interestingly, the efficiency 
of decoupling shows oscillatory behavior rather than a monotonic behavior. It has been noticed that the effectiveness of TPPM based sequences can be improved by combining the parent sequence into supercycles. The supercycled $\mathrm{SW}_{\mathrm{f}^{-}}$ TPPM-11 and -7 sequences give us enhanced decoupling. The trajectories of nuclear magnetization vector of the abundant nuclei in a simple spin system during TPPM and $\mathrm{SW}_{\mathrm{f}^{-}}$ TPPM decoupling were simulated on the Bloch sphere and compared with WALTZ-16, MLEV, and GARP decoupling sequences which are commonly used in liquids for removing heteronuclear couplings.

\section{Conflict of Interests}

The author declares that there is no conflict of interests regarding the publication of this paper.

\section{Acknowledgments}

The experiments discussed here are done at the Central NMR facility of Indian Institute of Technology Delhi, New Delhi, and the author is extremely grateful to Professor Narayanan Kurur for his fruitful suggestions in this project. Financial support from the University Grants Commission (UGC), India, is gratefully acknowledged.

\section{References}

[1] M. H. Levitt, Spin Dynamics: Basics of Nuclear Magnetic Resonance, John Wiley \& Sons, New York, NY, USA, 2001.

[2] R. Fu and G. Bodenhausen, "Evaluation of adiabatic frequencymodulated schemes for broadband decoupling in isotropic liquids," Journal of Magnetic Resonance. Series A, vol. 119, no. 1, pp. 129-133, 1996.

[3] M. H. Levitt and R. Freeman, "Composite pulse decoupling," Journal of Magnetic Resonance, vol. 43, no. 3, pp. 502-507, 1981.

[4] A. J. Shaka and J. Keeler, "Broadband spin decoupling in isotropic-liquids," Progress in Nuclear Magnetic Resonance Spectroscopy, vol. 19, no. 1, pp. 47-129, 1987.

[5] R. S. Thakur, N. D. Kurur, and P. K. Madhu, "Swept-frequency two-pulse phase modulation for heteronuclear dipolar decoupling in solid-state NMR," Chemical Physics Letters, vol. 426, no. 4-6, pp. 459-463, 2006.

[6] A. E. Bennett, C. M. Rienstra, M. Auger, K. V. Lakshmi, and R. G. Griffin, "Heteronuclear decoupling in rotating solids," The Journal of Chemical Physics, vol. 103, no. 16, pp. 6951-6958, 1995.

[7] R. S. Thakur, N. D. Kurur, and P. K. Madhu, "Improved heteronuclear dipolar decoupling sequences for liquid-crystal NMR," Journal of Magnetic Resonance, vol. 185, no. 2, pp. 264269, 2007.

[8] R. S. Thakur, N. D. Kurur, and P. K. Madhu, "An analysis of phase-modulated heteronuclear dipolar decoupling sequences in solid-state nuclear magnetic resonance," Journal of Magnetic Resonance, vol. 193, no. 1, pp. 77-88, 2008.

[9] R. S. Thakur, N. D. Kurur, and P. K. Madhu, "An experimental study of decoupling sequences for multiple-quantum and highresolution MAS experiments in solid-state NMR," Magnetic Resonance in Chemistry, vol. 46, no. 2, pp. 166-169, 2008.

[10] C. V. Chandran, P. K. Madhu, N. D. Kurur, and T. Bräuniger, "Swept-frequency two-pulse phase modulation $\left(\mathrm{SW}_{\mathrm{f}}\right.$-TPPM) sequences with linear sweep profile for heteronuclear decoupling in solid-state NMR," Magnetic Resonance in Chemistry, vol. 46, no. 10, pp. 943-947, 2008.

[11] C. V. Chandran and T. Bräuniger, "Efficient heteronuclear dipolar decoupling in solid-state NMR using frequency-swept SPINAL sequences," Journal of Magnetic Resonance, vol. 200, no. 2, pp. 226-232, 2009.

[12] C. V. Chandran, P. K. Madhu, P. Wormald, and T. Bräuniger, "Frequency-swept pulse sequences for 19F heteronuclear spin decoupling in solid-state NMR," Journal of Magnetic Resonance, vol. 206, no. 2, pp. 255-263, 2010.

[13] C. Augustine and N. D. Kurur, "Heteronuclear dipolar decoupling in liquid-crystal NMR using supercycled $\mathrm{SW}_{\mathrm{f}}$-TPPM sequences," Magnetic Resonance in Chemistry, vol. 48, no. 10, pp. 798-803, 2010.

[14] C. Augustine and N. D. Kurur, "Supercycled SW f $^{-T P P M}$ sequence for heteronuclear dipolar decoupling in solid-state nuclear magnetic resonance," Journal of Magnetic Resonance, vol. 209, no. 2, pp. 156-160, 2011.

[15] M. H. Levitt, R. Freeman, and T. Frenkiel, "Broadband heteronuclear decoupling," Journal of Magnetic Resonance, vol. 47, no. 2, pp. 328-330, 1982.

[16] M. H. Levitt, R. Freeman, and T. Frenkiel, "Supercycles for broadband heteronuclear decoupling," Journal of Magnetic Resonance, vol. 50, no. 1, pp. 157-160, 1982.

[17] M. H. Levitt, R. Freeman, and T. Frenkiel, "Broadband decoupling in high-resolution nuclear magnetic resonance spectroscopy," Advances in Magnetic and Optical Resonance, vol. 11, pp. 47-110, 1983.

[18] A. J. Shaka, J. Keeler, T. Frenkiel, and R. Freeman, "An improved sequence for broadband decoupling: WALTZ-16," Journal of Magnetic Resonance, vol. 52, no. 2, pp. 335-338, 1983.

[19] A. J. Shaka, J. Keeler, and R. Freeman, "Evaluation of a new broadband decoupling sequence: WALTZ-16," Journal of Magnetic Resonance, vol. 53, no. 2, pp. 313-340, 1983.

[20] A. J. Shaka, P. B. Barker, and R. Freeman, "Computer-optimized decoupling scheme for wideband applications and low-level operation," Journal of Magnetic Resonance, vol. 64, no. 3, pp. 547-552, 1985.

[21] Y. Yu and B. M. Fung, "An efficient broadband decoupling sequence for liquid crystals," Journal of Magnetic Resonance, vol. 130, no. 2, pp. 317-320, 1998.

[22] B. M. Fung, A. K. Khitrin, and K. Ermolaev, "An improved broadband decoupling sequence for liquid crystals and solids," Journal of Magnetic Resonance, vol. 142, no. 1, pp. 97-101, 2000. 

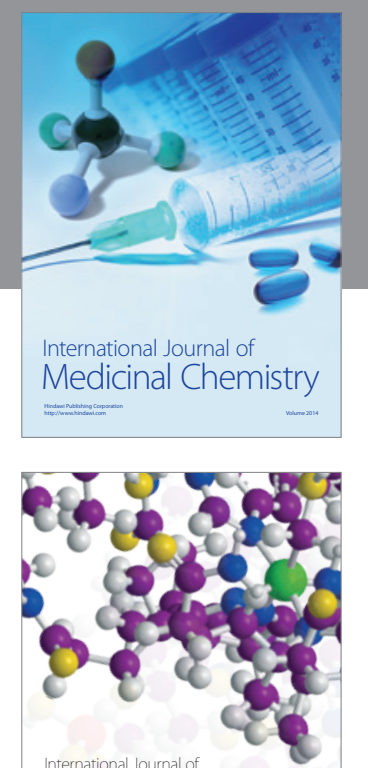

\section{Carbohydrate} Chemistry

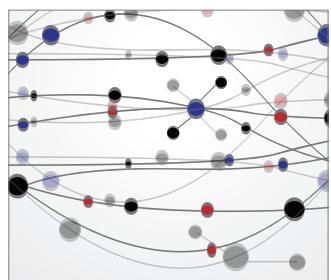

The Scientific World Journal
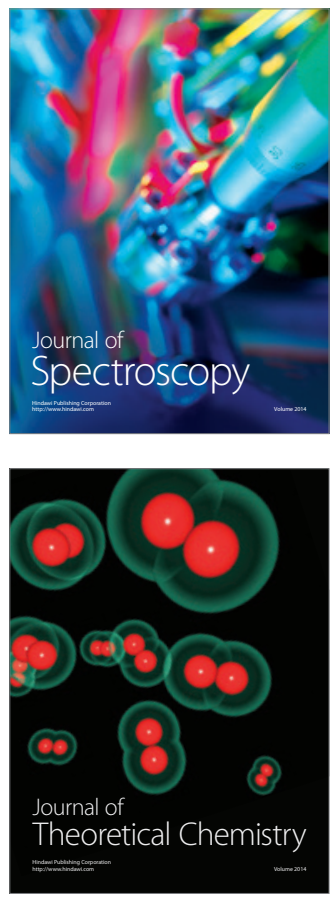
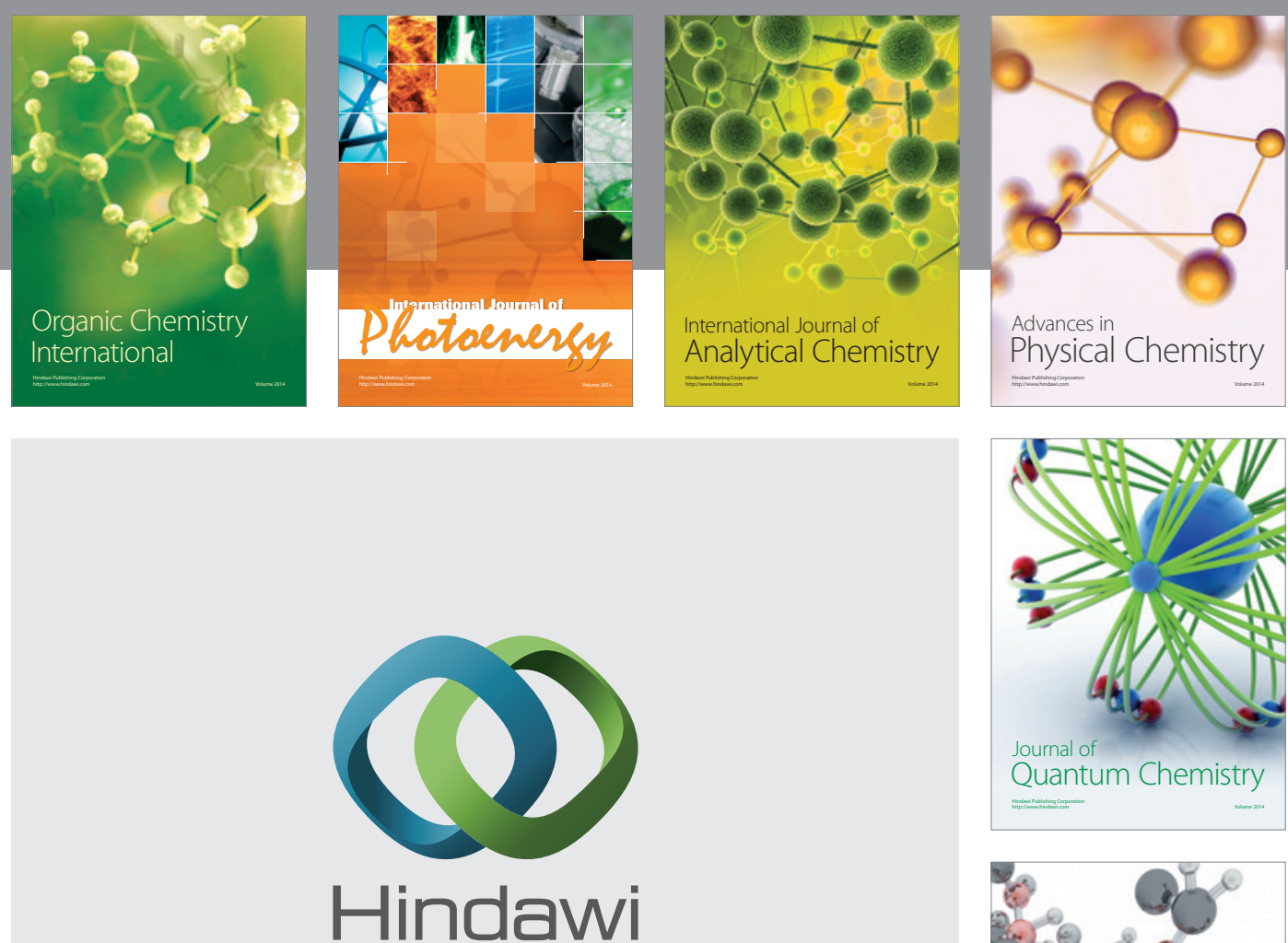

Submit your manuscripts at

http://www.hindawi.com

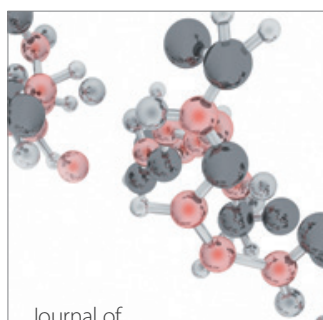

Analytical Methods

in Chemistry

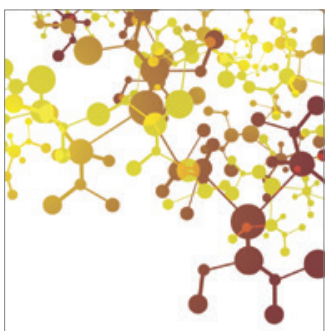

Journal of

Applied Chemistry

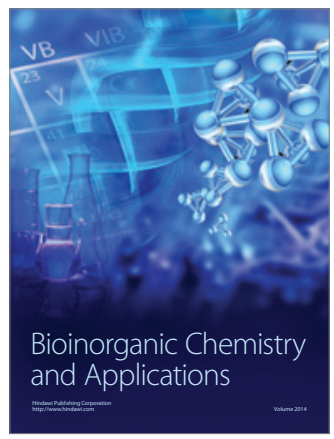

Inorganic Chemistry
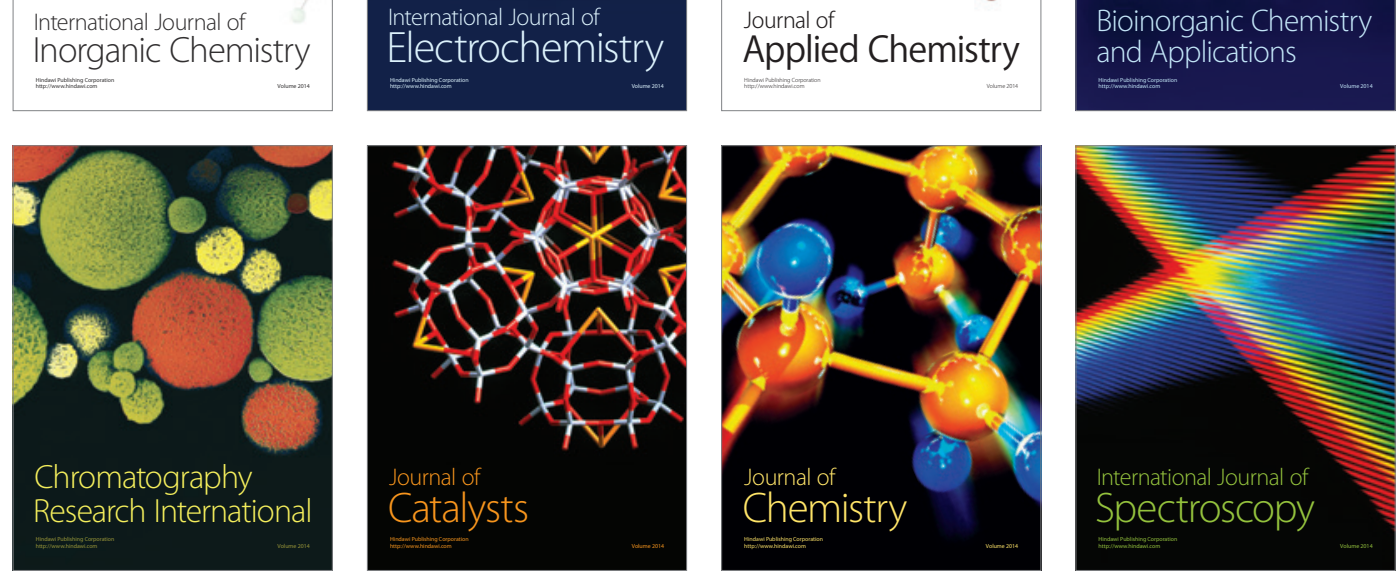